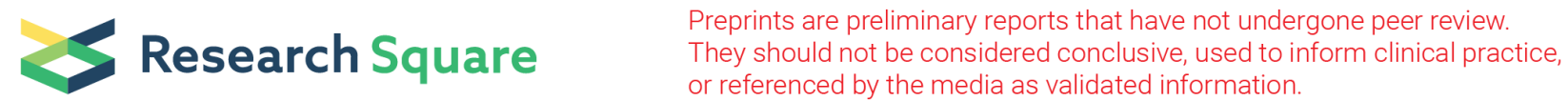

\section{Sclerosing Angiomatoid Nodular Transformation of the Spleen, a Rare Cause for Laparoscopic Splenectomy: a Case Report and Literature Review}

Jianyang Yang

Southern Medical University

Yue Zhang

Shenzhen people's hospital

Gongze Peng

Shenzhen people's hospital

Tianchong Wu ( $\square$ drwutianchong@sina.com )

Shenzhen People's Hospital

\section{Case report}

Keywords: SANT, laparoscopic splenectomy, case report

Posted Date: December 30th, 2020

DOI: https://doi.org/10.21203/rs.3.rs-136193/v1

License: @ (i) This work is licensed under a Creative Commons Attribution 4.0 International License.

Read Full License 


\section{Abstract}

BACKGROUND: Sclerosing angiomatoid nodular transformation of the spleen is one kind of exceedingly rare benign lesions originated from spleen. The incidence and pathogen remain unclear partially due to its rarity and short history. Consequently, preoperative diagnosis become extremely perplex. Splenectomy is a predominated therapy approach as well as diagnosis method for sclerosing angiomatoid nodular transformation of the spleen. We reported a case underwent computed tomography scan, magnetic resonance imaging and ultrasonic scan preoperatively. Examinations conclusion highly suspected sclerosing angiomatoid nodular transformation of the spleen and was confirmed by pathology after laparoscopic splenectomy. We herein reviewed some literatures on imaging characters of to reveal the possibility of preoperative diagnosis when clinicians encounter and investigated whether laparoscopic splenectomy is an appropriate treatment.

CASE PRESENTATION: A 50-year-old female was found with a low dense mass in the spleen by abdominal computed tomography incidentally without any positive symptom. Further enhanced computed tomography scan revealed the lesion with asynchronous enhancement and magnetic resonance imaging showed radio-like rift missing from central. Ultrasonic scan imaging presented hypoechoic gap in the center, pretty similar to magnetic resonance imaging. These radiology imaging features deduced to Sclerosing angiomatoid nodular transformation of the spleen. Laparoscopic splenectomy was performed successfully by our team and histopathological findings convinced the preoperative diagnosis. Gross inspection was coincident to the performance of magnetic resonance imaging.

CONCLUSION: By comprehending imaging character profoundly, sclerosing angiomatoid nodular transformation of the spleen can be a differential diagnosis preoperatively when encounter lesion of spleen. We believe that laparoscopic splenectomy is a reliable and effective therapy option for such rare benign lesion of spleen without risk of recurrence.

\section{Background}

Sclerosing angiomatoid nodular transformation (SANT) is a rare benign lesion originated in the spleen with unclear pathogen. It's a very "young" lesion in spleen because of its short history. Martel et al. firstly bring the concept of SANT in the medicine by summarized 25 cases pathology characteristic of SANT in 2004 [1]. With only several hundred cases of SANT have been reported up-to-date, most of physicians, surgeons, and radiologists still lack acknowledge of SANT owing to its rarity. SANT is usually mistaken as splenic inflammatory pseudotumor, hamartoma, or even malignant tumor when encounter spleen lesion in clinic [2]. Splenectomy is rather a method to reaches diagnosed than treatment in most cases because it's very difficult to diagnose SANT preoperatively $[3,4]$. Therefore, we reported a case of SANT diagnosed preoperatively and treated by laparoscopy splenectomy in the end. We reviewed literature to investigate the preoperative diagnose of SANT and the appropriate treatment for SANT. 


\section{Case Presentation}

A 50-year-old Hakka female was found with a low dense mass in the spleen by abdominal computed tomography (CT) during health checkup. She was asymptomatic and didn't have any disease history. There were no positive signs found in abdominal physical examination. Parameters of blood routine, liver and kidney functions tests and serum tumor markers were unremarkable. For further treatment, patient was hospitalized in our department. On the abdominal non-enhanced plain CT scan, a lesion was faintly observed with a vague margin, whose Hounsfield unit was approximate 41, while the surrounding normal splenic tissue were 43(Fig. 1A). However, it was showed up significantly as a weakly enhanced lobulatedlike mass of $57 \times 41 \times 50 \mathrm{~mm}$ with clear boundary on CT plain scan during rapid enhancement period. Compared to the enhancement of surrounding normal spleen $(205 \mathrm{HU})$, the mass presented heterogeneous as well as much lower enhancement with a value of $69 \mathrm{HU}(\mathrm{Fig}$. 1B). The CT value of the mass progressively increased to the peak of $127 \mathrm{Hu}$ on portal venous enhancement and delayed enhanced period, but still lower than that of the surrounding spleen (134HU). (Fig. 1C and 1D). Our radiologists proposed diagnosis of SANT differential from other benign lesion in the spleen. Sequently, she underwent an abdominal magnetic resonance imaging (MRI). The lesion showed an iso-intensity to the normal spleen on T1 -weighted (MRI) (Fig. 2A) but a little lower intensity in the T2-weighted (Fig. 2B). The lesion appeared heterogenous hypo-intensity with lower intensity radial-like rift emission from central on T2-weighted, especial on fat-suppressed (Fig. 2A and 2B). The mass was progressively enhanced during MRI enhanced phased and eventually surpass the background spleen (Fig. 2C凶2D and 2F). The conclusion of MRI also highly recommended SANT as first diagnose. Interestingly, radial-like hypoechoic gap separating mass also showed up on ultrasonic scan. (Fig. 3)

To get a precise pathologic diagnose, laparoscopic splenectomy was successfully operated under patient's authorize. As is showed in the Fig. 4.A, the resected specimen appears as a well-defined mass encircled by multiple nodules which are wrapped in transparent shell. And there's a characteristic white stellate colloid in the central of lesion. At low magnification, there are a great number of slit-like, round, or irregular shaped vascular nodules deformed by dense collagen fibrous or fibrosclerotic stroma. Plentiful erythrocytes and stromal cells with mild chronic inflammatory cell infiltration can be observed within the nodules under high magnification. Immunohistochemical staining of specimen presented three typical types vessels directly pointing to the SANT diagnose, which are (1)sinusoid type, both CD8 and CD31 positive but CD34negative vessels;(2) small vein type, CD31positive but CD8 and CD34negative vessels; and (3) cord capillary type, both CD34 and CD31 positive but CD8negative vessels.

There is no any complication and no sign of recurrence so far.

\section{Discussion And Conclusion}

SANT is a solitary entity distinguishing from other splenic vascular tumors and firstly defined by Martel et al because of its unique pathology [1]. The incidence of SANT remains unknown due to its rarity. In previous literatures, SANT mainly occurs in middle-aged population with a slightly female inclination and 
the mean age at onset was 50 years, which is also supported in our case [5]. However, occurrence of SANT shows a young age trends in recent report [6, 7]. Youngest SANT patient was a 7-month-old infant with splenomegaly [8]. Most cases are found incidentally since most patients are asymptomatic. Majority symptomatic patients attended to clinic with main complaints of abdominal discomfort, others were nonspecific such as flank pain, weight loss, fatigue, night sweat, and mild fever (9).

With increasing cases reported, radiologist and physicians tried to conclude imaging characters of SANT in order to get preoperative diagnoses. On non-enhanced plain CT scan, SANT presented as hypodense mass predominantly, some as equal-density, and partial cases have small central calcification [10]. Further enhanced CT presented solitary well- circumscribed hypodensity lesion comparing to background spleen in artery phased. The contrast between lesion with parenchyma of the spleen was significant in rapid enhanced phased and diminished progressively in portal-venous phased and delayed phased [11]. Karaosmanoglu et al firstly report imaging on MRI, which presented as central hyperintense area simulating hemorrhage on T1-weighted MRI. What's more, they described the enhancement of SANT as progressively centripetal infiltration and the specific pattern was vividly named as "spoke-and-wheel" [12]. In Jianbing Ma's literature, "spoke-and-wheel" pattern in CT or MRI examination can be the key for SANT diagnoses with $58 \%$ occurrence rate in total 12 patients [13]. However, only 4 of 12 cases were diagnosed as SANT preoperatively indeed probably due to its rarity. Profound research on correlation between pathological and radiological characters demonstrated that spoke-and-wheel imaging is referenced to a stellate collagen stroma core surrounded by angiomatoid nodules that are separated with proliferative fibrous septa [10]. As is shown in Table1, there is few preoperative diagnoses cases reported and we were the one of several cases. In presented case, SANT was nearly iso-density in both non-enhanced CT and pre-contrast T1 $10 \mathrm{~T} 2$ weighted on MRI, and T2 weighted could observed satellite hypodensity septa in center. The multiple phase imaging of CT was consistent with previous report, which was enhanced progressively but remain hypodensity to background. Although spoke-and-wheel was not presented, central satellite hypodensity septa existed lasting the whole enhancement of T1 weighted on MRI. By summarized previous literature, we repute that there are two typical types of enhanced imaging characters on CT and MRI:1) enhancement started from peripheral part in progressively way presenting as spoke-and-wheel pattern; 2) enhancement evaluated progressively in entity way with or without hypointensity septa in center. Our case belongs to the later one. Similar to spoke-and wheel pattern, we suppose that satellite hypo-intensity septa are also corresponded to the colloid scar in center of gross. Another features we learned is that the CT value of SANT remain lower to the background of spleen during multiple phase. We believe that these features are helpful in preoperative diagnosis when spokeand-wheel sign was absent.

Gray-scale ultrasonography usually shows a heterogeneously hypoechoic or isoechoic mass with hypoechoic rim compared to the surrounding splenic parenchyma. The lesion appeared "spoke-andwheel" enhancement pattern as well on contrast sonography [14, 15]. Our case presented as heterogeneously majority isoechoic mass with hypoechoic radio-like linear area in center, which is similar to T2-weighted on MRI (Fig. 3 and Fig. 2A). 
Positronemission tomographyCT(PET-CT) is a powerful examination mainly applying in malignant tumor follow-up. SANT presented slight FDG accumulation, similar to tumor metastasis. John et. concluded that SANT can be a differential diagnosis in the patient with malignant tumor history $[16,17]$.

Needle biopsy possesses excellent accuracy in preoperative diagnose despite accompanying with the risks of bleeding and needle path metastasis. Literature shows that bleeding risk of spleen needle biopsy can be retained in very low level under the ultrasonic guidance $[16,18]$.

Laparoscopic splenectomy is a prevalent therapy approach for variety spleen benign lesions, in which SANT accounted for 1.7\%[4]. In our case, laparoscopic splenectomy was implemented in consideration that SANT is a benign mass and laparoscopic surgery has been surgery mature as well as regular in our department. In a series study with 37 cases, laparoscopic partial splenectomy demonstrated a great advantage in the sequent complication of surgery compare to total splenectomy [19].

Pathology is gold standard for SANT diagnosis. The gross appearance of resected spleen is coincided with spoke-wheel pattern in CT and MRI imaging, which presented as a wellcircumscribed mass with a stellate white stroma at the center [10]. Under microscope, classic pathological characters are as follow:i) multiple angiomatoid nodules are overspread in the horizon separated by dense collagen fibrosis and fibroid rims. ii)Individual nodule is composed of slit-shaped, round, or irregularly shaped lumens lined by swollen endothelial cells. iii)numerous red blood cells with scattered inflammatory cells are crowded in the inflammatory cells. iv)Three typical types blood vessels are presented with immunohistochemical staining cord capillary-like type with CD34 $\triangle \mathrm{CD} 31$ co-expression and absence of CD8-, sinusoids-like type (CD34-/CD8+/CD31+), and small veins-like type (CD34-/CD8-/CD31+) $[1,20]$. Those features mentioned above can be found in our case. Additionally, some researchers detected plenty IgG4-positive plasma cells that and proposed SANT as an IgG4 related disease [21,22]. But the hypothesis was not approved by advanced studies, in which cheng et al revealed that SANT is a reactive lesion originated polyclonally rather than a neoplasm [23]. What's more, no cases associated with IgG4 disease has been reported up to date.

In summary, SANT is potential to be diagnosed preoperatively when imaging features are profoundly comprehended. Laparoscopic splenectomy is a reliable and effective therapy option for SANT treatment or diagnosis.

\section{Abbreviations}

Sclerosing angiomatoid nodular transformation: SANT; computed tomography: CT; Hounsfield unit: HU; magnetic resonance imaging: MRI; fine needle aspiration: FNA; Ultrasonography: US;

\section{Declarations}

\section{Ethics approval and consent to participate}


The Ethics Committee of The Second Medical College of Jinan University, Shenzhen People's Hospital approved this study. The patient signed an informed consent form for surgery.

\section{Consent for publication}

Not applicable.

\section{Availability of data and materials}

The datasets used or analyzed in the current study are available from the corresponding author on reasonable request.

\section{Competing interests}

The authors declare that they have no competing interests.

\section{Funding}

This work was supported by Science and Technology Innovation Foundation of Shenzhen [grant number JCYJ20180228164603659] and [grant number JCYJ20180507182437217].

\section{Authors' contributions}

TCW, YZ, and GZP conducted the surgery. TCW and YZ designed the report. YJY collected patient's clinical data and information. YJY and GZP wrote the paper. All authors read and approved the final manuscript.

\section{Acknowledgements}

The authors wish to thank the department of anesthesiology of Shenzhen People's Hospital for their superb anesthetic technique.

\section{References}

1. Martel M, Cheuk W, Lombardi L, Lifschitz-Mercer B, Chan JK,Rosai J. Sclerosing angiomatoid nodular transformation (SANT):report of 25 cases of a distinctive benign splenic lesion. Am J Surg Pathol. 2004;28(10):1268-79.

2. Önder S, Kosemehmetoglu K, Himmetoglu Ç, Firat P, Uner A. Sclerosing angiomatoid nodular transformation (SANT) of spleen: a case report describing cytology, histology, immunoprofile and differential diagnosis.; Cytopathology. 2012;23(2):129-32.

3. Gonzalez-Urquijo M, Rodarte-Shade M, Gil-Galindo G. Splenic Primary Solid Tumors : Does a Preoperative Histopathology Diagnosis Really Matter? Am Surg. 2020 16;3134820951480.

4. Raman SP, Singhi A, Horton KM, Hruban RH, Fishman EK. Sclerosing angiomatoid nodular transformation of the spleen (SANT): multimodality imaging appearance of five cases with 
radiology-pathology correlation. ABDOM IMAGING. 2013;38(4):827-34.

5. Wang T, Hu B, Liu D, Gao Z, Shi H, Dong W. Sclerosing angiomatoid nodular transformation of the spleen: A case report and literature review. ONCOL LETT. 2016;12(2):928-32.

6. Zhang S, Yang W, Hongyan XU, Zhuqiang WU. Sclerosing Angiomatoid Nodular Transformation of Spleen in a 3-year-old Child.; Indian Pediatr. 2015;52(12):1081-3.

7. Jamal A, Siddique U, Inam Ur Rehman S, Arain M, Hamid A. Sclerosing angiomatoid nodular transformation of spleen in a 8-year old girl. J Pak Med Assoc. 2020 70(8):1448-1450.

8. Pelizzo G, Villanacci V, Lorenzi L, Doria O, Caruso AM, Girgenti V, Unti E, Putignano L, Bassotti G, Calcaterra V. Sclerosing angiomatoid nodular transformation presenting with abdominal hemorrhage: First report in infancy. Pediatric reports. 2019;11(2):7848.

9. Nomura R, Tokumura H, Katayose Y, Nakayama F, Iwama N, Furihata M. Sclerosing Angiomatoid Nodular Transformation of the Spleen: Lessons from a Rare Case and Review of the Literature.,Intern Med. 2019;58(10):1433-1441.

10. Lewis RB, Lattin GE Jr, Nandedkar M ,Aguilera NS.Sclerosing angiomatoid nodular transformation of the spleen: CT and MRI features with pathologic correlation.AJR Am J Roentgenol. 2013 Apr;200(4):W353-60.

11. Thipphavong S, Duigenan S, Schindera ST, Gee MS, Philips S. Nonneoplastic, benign, and malignant splenic diseases: crosssectional imaging findings and rare disease entities. AJR Am J Roentgenol. 2014 Aug;203(2):315-22.

12. Karaosmanoglu DA, Karcaaltincaba M, Akata D. CT and MRI findings of sclerosing angiomatoid nodular transformation of the spleen: spoke wheel pattern. Korean J Radiol 2008;9(suppl): S52-5.

13. Ma J, Zhang W, Wang L, Zhu Z, Wang J, Zhang J, Yang X. Imaging Features of Sclerosing Angiomatoid Nodular Transformation in Spleen. J COMPUT ASSIST TOMO. 2019;43(6):863-9.

14. Gutzeit A, Stuckmann G, Dommann-Scherrer C. Sclerosing angiomatoidvnodular transformation (SANT) of the spleen: sonographic finding.J Clin Ultrasound. 2009 37(5):308-11.

15. Kim HJ, Kim KOW, Yu ES, Byun JH, Lee SS, Kim JH, et al.Sclerosing angiomatoid nodular transformation of the spleen: clinical and radiologic characteristics. Acta Radiol. 2012 53: 701-706, 1.

16. Matsubara K, Oshita A, Nishisaka T, Sasaki T, Matsugu Y, Nakahara H, Urushihara T, Itamoto T. A case of sclerosing angiomatoid nodular transformation of the spleen with increased accumulation of fluorodeoxyglucose after 5-year follow-up. Int J Surg Case Rep. 2017;39:9-13

17. Sharma P. 18F-FDG avid Sclerosing Angiomatoid Nodular Transformation (SANT) of spleen on PETCT - a rare mimicker of metastasis.; Nucl Med Rev Cent East Eur. 2018;21(1):53.

18. Mclnnes MD, Kielar AZ, Macdonald DB. Percutaneous image-guided biopsy of the spleen: systematic review and meta-analysis of the complication rate and diagnostic accuracy. Radiology. 2011;260(3):699-708. 
19. Jin Y, Hu H, Regmi P, Li F, Cheng N. Treatment options for sclerosing angiomatoid nodular transformation of spleen. HPB: the official journal of the International Hepato Pancreato Biliary Association. 2020;22(11):1577-1582.

20. Sangiorgio VFI, Arber DA. Non-hematopoietic neoplastic and pseudoneoplastic lesions of the spleen. Semin Diagn Pathol. 2020;S0740-2570(20)30061-7.

21. Kuo TT, Chen TC, Lee LY. Sclerosing angiomatoid nodular transformation of the spleen (SANT): clinicopathological study of 10 cases with or without abdominal disseminated calcifying fibrous tumors, and the presence of a significant number of IgG4+ plasma cells. Pathol Int. 2009;59(12):84450 .

22. Nagai $Y$, Hayama N, Kishimoto T, Furuya M, Takahashi Y, Otsuka M, et al. Predominance of IgG4+ plasma cells and CD68 positivity in sclerosing angiomatoid nodular transformation (SANT). Histopathology. 2008;53(4):495-8.

23. Chang KC, Lee JC, Wang YC, Hung LY, Huang Y, Huang WT, et al. Polyclonality in Sclerosing Angiomatoid Nodular Transformation of the Spleen. Am J Surg Pathol. 2016;40(10):1343-51.

\section{Tables}

Table 1

Summary of preoperative SANT diagnosis in previous reports

\begin{tabular}{|lllllll|}
\hline Author & $\begin{array}{l}\text { Case } \\
\text { number }\end{array}$ & Age & Diameter & $\begin{array}{l}\text { Preoperative } \\
\text { Diagnosis }\end{array}$ & $\begin{array}{l}\text { Type of } \\
\text { examination }\end{array}$ & treatment \\
\hline $\begin{array}{l}\text { Jianbing } \\
\text { Ma }\end{array}$ & 4 & $\begin{array}{l}21- \\
62^{*}\end{array}$ & $30-85^{*}$ & SANT & CT,MRI & splenectomy \\
$\begin{array}{l}\text { Keiso } \\
\text { Matsubara }\end{array}$ & 1 & 64 & $4.5 \mathrm{~cm}$ & SANT & $\begin{array}{l}\text { CT,MRI,PET- } \\
\text { CT }\end{array}$ & $\begin{array}{l}\text { Follow up for } 5 \text { years, } \\
\text { splenectomy eventually }\end{array}$ \\
\hline $\begin{array}{l}\text { Punit } \\
\text { Sharma }\end{array}$ & 1 & 56 & $3.9 \mathrm{~cm}$ & SANT & PET-CT,FNA & Follow up \\
\hline $\begin{array}{l}\text { Present } \\
\text { case }\end{array}$ & 1 & 50 & $4.5 \mathrm{~cm}$ & SANT & CT,MRI & splenectomy \\
\hline *no mention in detial & & & & & \\
\hline
\end{tabular}

\section{Figures}




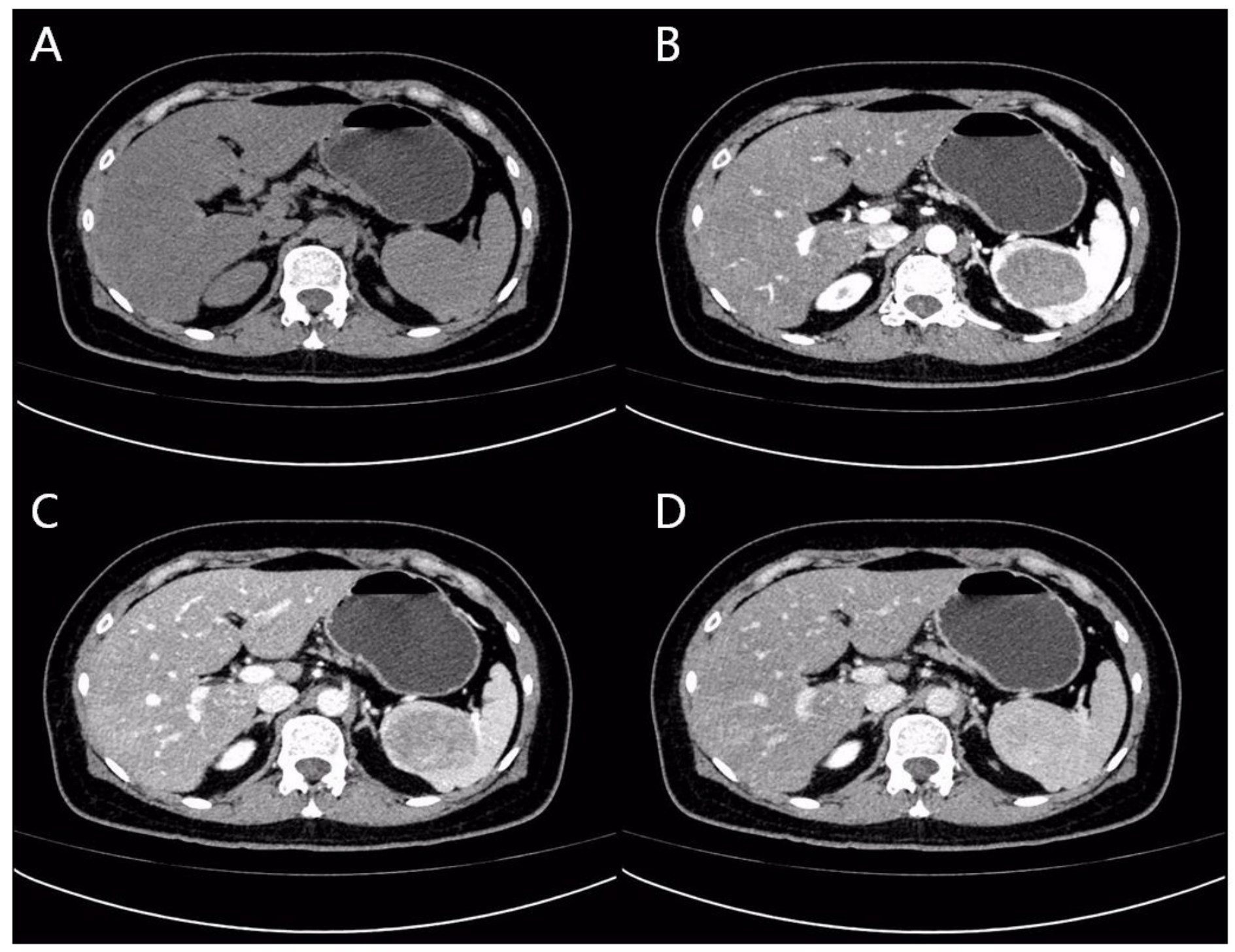

Figure 1

SANT showed asynchronous enhancement comparing to parenchyma of spleen on CT scan multiple phase. A, non-enhanced CT scan $\triangle B$, artery enhanced period CT scan囚 $\triangle$, portal venous enhanced period CT scan $₫ \mathrm{D}$, delayed enhanced period CT scan. 


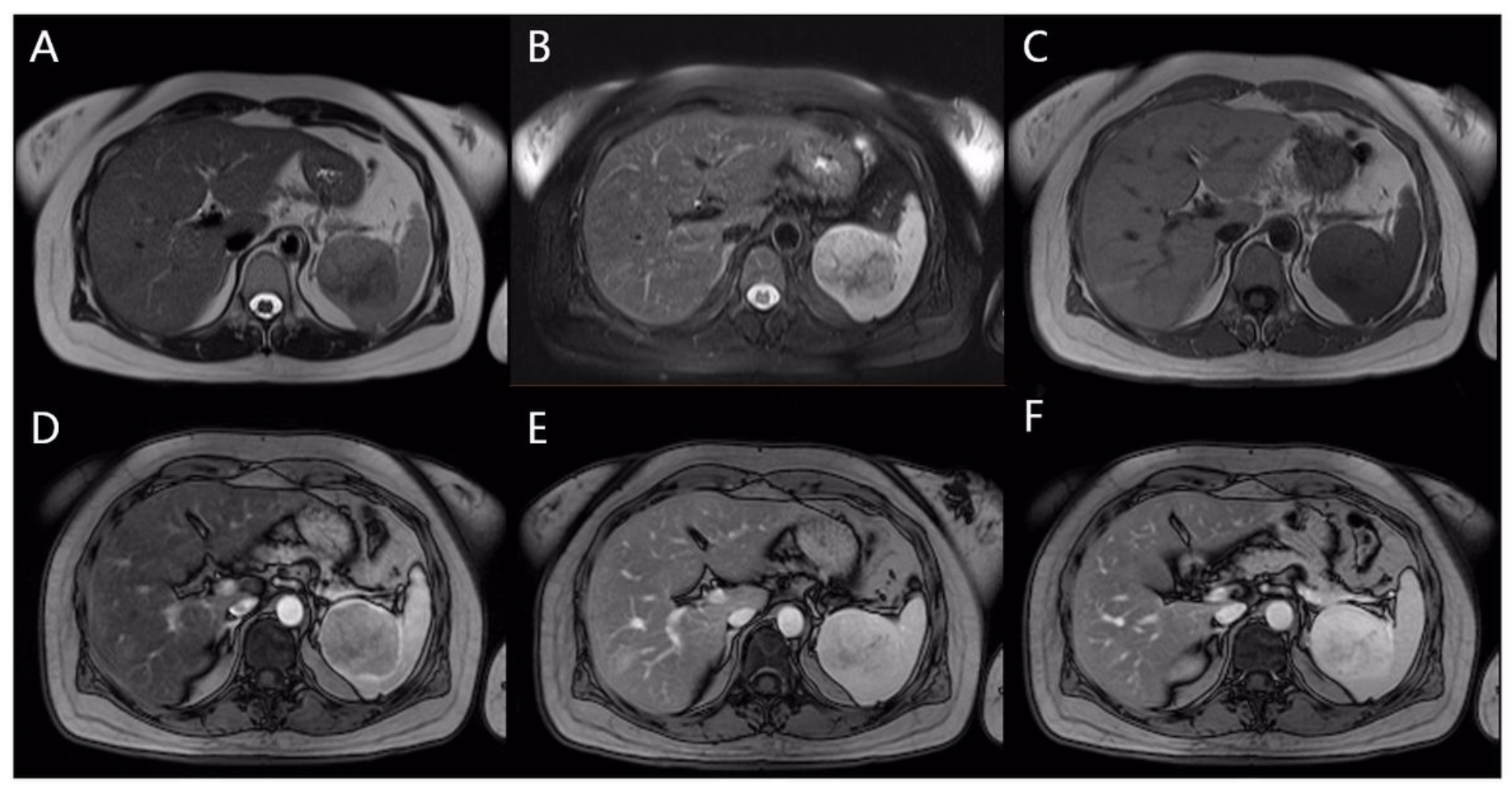

\section{Figure 2}

SANT presented radio-like hypointensity rift splitting in central on MRI multiple phases. A, T2-weighted MRI on fat-saturate; B, T2-weighted MRI on fat-suppress ; C, T1-weighted MRI of precontrast; D-E, T1weighted MRI after injection 


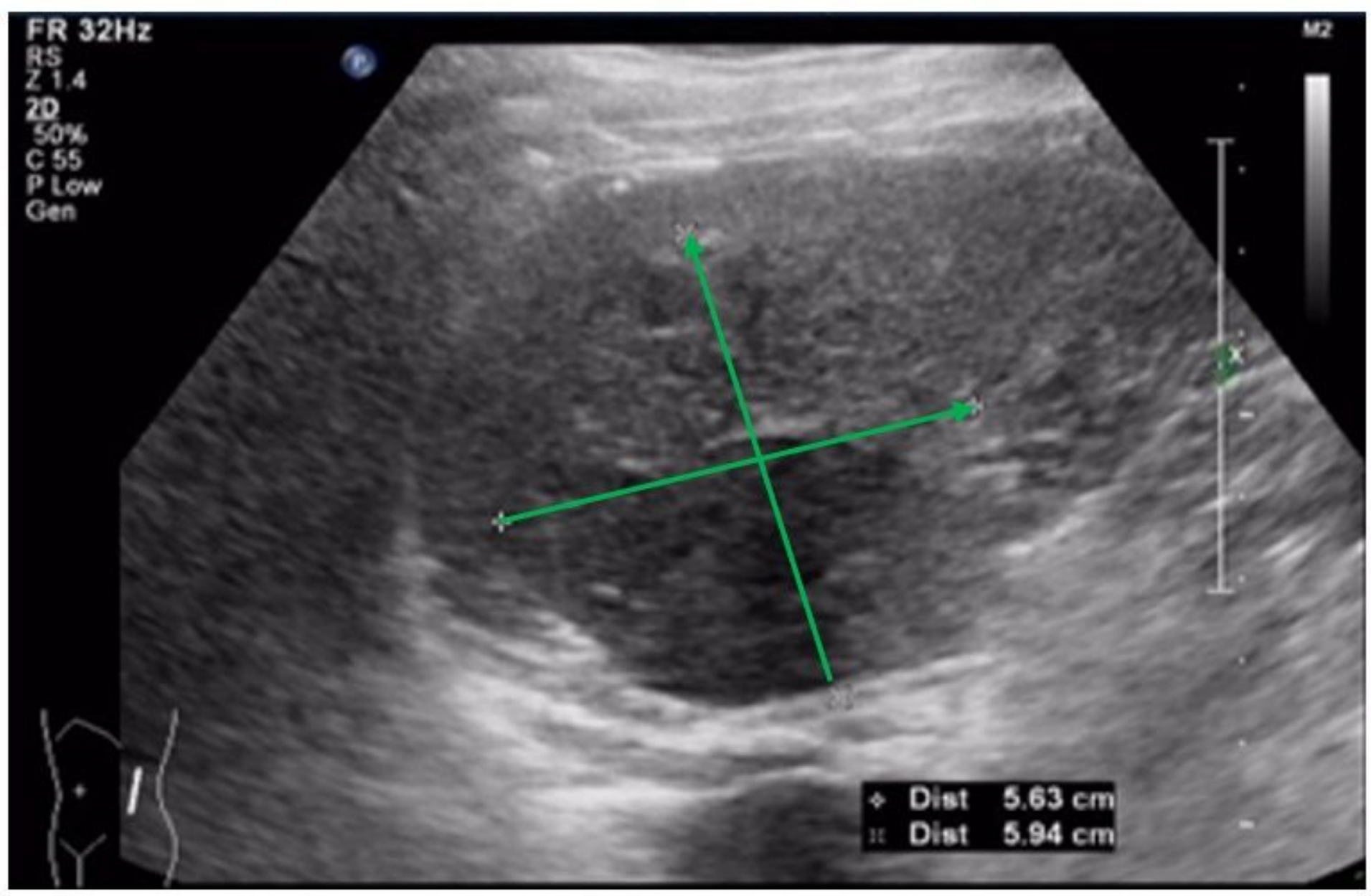

Figure 3

Imaging of SANT on US 


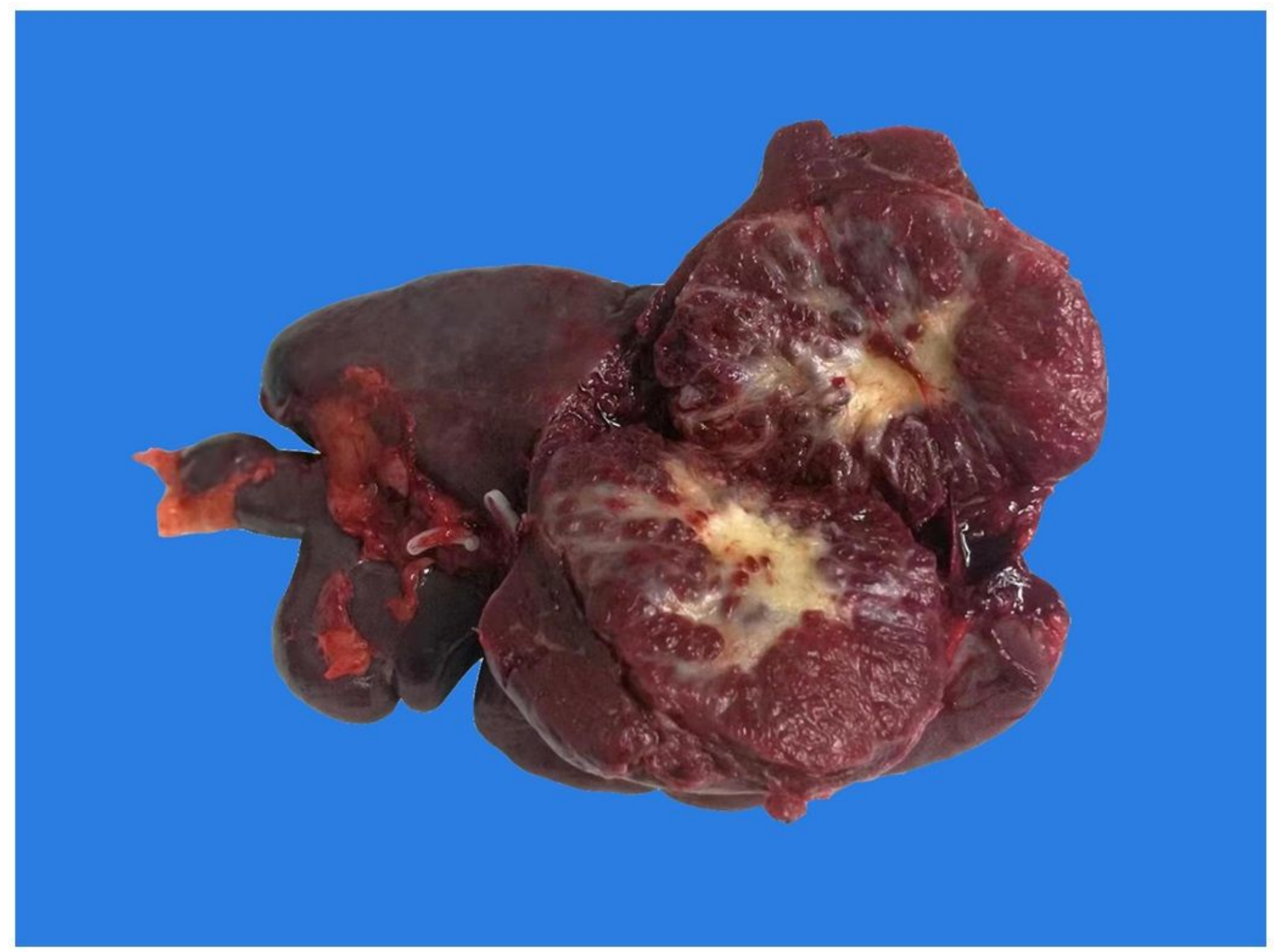

\section{Figure 4}

Macroscopic examination of the postoperative specimen. The resected lesion measured $5.8 * 4.6 * 4.3 \mathrm{~cm}$ in size 